\title{
CONVERGENCE AND REMAINDER TERMS IN LINEAR RANK STATISTICS ${ }^{1}$
}

\author{
By Harald Bergström and Madan L. Puri \\ University of Göteborg, Chalmers University of Technology, \\ and Indiana University
}

\begin{abstract}
A new approach to the asymptotic normality of simple linear rank statistics for the regression case studied earlier by Hájek (1968) is provided along with the estimation of the remainder term in the approximation to normality.
\end{abstract}

1. Introduction and summary. Let $X_{1}, \ldots, X_{n}$ be independent random variables having continuous cdf's (cumulative distribution functions) $F_{1}(x), \ldots$, $F_{n}(x)$ respectively. Consider a statistic $S_{n}=s\left(X_{1}, \ldots, X_{n}\right)$ with $E S_{n}=0$ and $E S_{n}{ }^{2}<\infty$. Then, to prove the asymptotic normality of $S_{n}$ (as $\left.n \rightarrow \infty\right)$, Hájek (1968) uses the method of projection which gives to the statistic $S_{n}$, the approximation of the form

$$
\hat{S}_{n}=\sum_{j=1}^{n} E\left[S_{n} \mid X_{j}\right] .
$$

Consider now the simple linear rank statistic $S_{n}$ introduced by Hájek (1962, 1968)

$$
S_{n}=\sum_{j=1}^{n} c_{j}\left\{\psi\left(R_{j} / n\right)-E\left[\psi\left(R_{j} / n\right)\right]\right\}
$$

where the $c$ 's are known constants, $R_{j}$ is the rank of $X_{j}$ among $\left(X_{1}, \ldots, X_{n}\right)$ and $\psi(\cdot)$ is a score generating function defined on $(0,1)$. Hájek $(1962)$ [see also Hájek-Šiák (1967)] established the asymptotic normality of $S_{n}$ in (1.2) under the assumption that the $F_{i}$ are contiguous, e.g., when $F_{i}(x)=F\left(x-\Delta d_{n i}\right)$ where $\Delta$ is the unknown parameter and the $d$ 's are the known constants. Later on Hájek (1968) studied the asymptotic normality of $S_{n}$ for the general $F_{i}(x)$ (the noncontiguous case). Under the setup of Hájek (1962), Jurečková and Puri (1975), referred to hereafter as JP, studied the problem of determining the rate of convergence of the cdf of $S_{n}$ to the limiting normal cdf and established it of order $O\left(N^{-\frac{1}{2}+\delta}\right)$ for $\delta>0$. In this paper we not only give a new approach to the asymptotic normality of $S_{n}$ for the general $F_{i}$ (i.e., not necessarily contiguous) but improve the results of JP in providing a sharper bound (for the general $F_{i}$ 's). In the passing, we may also mention that whereas JP requires $\phi$ to have a bounded fourth derivative, here we only require the boundedness of the second

Received May 1975; revised January 1977.

${ }^{1}$ Work supported by the Air Force Office of Scientific Research, AFSC, USAF, under Grant No. AFOSR 76-2927. Reproduction in whole or in part is permitted for any purpose of the U.S. Government. Part of this work was done while the author was the recipient of a Senior U.S. Scientist Award from the Alexander-von-Humboldt-Foundation.

AMS 1970 subject classifications. Primary 62E20; Secondary 60F05, 60F99.

Key words and phrases. Linear rank statistics, score generating functions, rate of convergence. 
derivative. Furthermore whereas this paper gives more explicit error bounds than the JP paper, the latter gives more information on the limiting behavior of $E S_{n}$ and $\operatorname{Var} S_{n}$.

We now introduce some notations. We define $\psi(\cdot)=0$ outside $(0,1)$. Then, we can use the supremum norm

$$
\|\psi\|=\sup _{t \in(-\infty, \infty)}|\psi(t)| .
$$

Set

$$
\begin{aligned}
& \rho_{i}=R_{i} / n, \quad \rho_{i i}=E\left[\rho_{i} \mid X_{i}\right], \quad u(x)=1 \quad \text { if } \quad x \geqq 0 \\
& \text { and } \quad u(x)=0 \text { otherwise. }
\end{aligned}
$$

Then

$$
R_{i}=\sum_{j=1}^{n} u\left(X_{i}-X_{j}\right) .
$$

In this paper, we shall deal with the following approximation of $S_{n}$.

$$
T_{n}=\sum_{i=1}^{n} c_{i}\left\{\psi\left(\rho_{i i}\right)-E\left[\psi\left(\rho_{i i}\right)\right]+\left(\rho_{i}-\rho_{i i}\right) \psi^{\prime}\left(\rho_{i i}\right)\right\},
$$

assuming that $\psi^{\prime}$ exists on $(0,1)$ and

$$
\hat{T}_{n}=\sum_{j=1}^{n} E\left[T_{n} \mid X_{j}\right] .
$$

Since $E\left[\left(\rho_{i}-\rho_{i i}\right) \psi^{\prime}\left(\rho_{i i}\right)\right]=0$, it follows that

$$
\hat{T}_{n}=\sum_{i=1}^{n} c_{i}\left\{\psi\left(\rho_{i i}\right)-E\left[\psi\left(\rho_{i i}\right)\right]+\sum_{j \neq i}^{n} E\left[\left(\rho_{i}-\rho_{i i}\right) \dot{\psi}^{\prime}\left(\rho_{i i}\right) \mid X_{j}\right]\right\} .
$$

Let $H_{n}, G_{n}$ and $\hat{G}_{n}$ be the cdf's of $S_{n}, T_{n}$ and $\hat{T}_{n}$ respectively, and put

$$
\sigma_{n}{ }^{2}=E\left[S_{n}{ }^{2}\right], \quad \hat{\delta}_{n}{ }^{2}=E\left[\hat{T}_{n}{ }^{2}\right], \quad \Gamma_{n r}^{2 r}=\frac{1}{n} \sum_{i=1}^{n} c_{i}{ }^{2 r}, \quad \Gamma_{n r}>0 .
$$

Then our theorems are the following:

THEOREM 1.1. If $\psi$ has a derivative on $(0,1)$ then

$$
\begin{aligned}
& \left\|\hat{G}_{n}\left(\hat{\delta}_{n} \cdot\right)-\Phi(\cdot)\right\| \leqq 4 C\left[2\|\phi\|^{3}+\left\|\psi^{\prime}\right\|^{3}\right] \sum_{i=1}^{n}\left|c_{i}\right|^{3} \hat{\delta}_{n}^{-3} ; \\
& \Phi(x)=(2 \pi)^{-\frac{1}{2}} \int_{-\infty}^{x} e^{-t 2 / 2} d t
\end{aligned}
$$

where $C$ is the constant in Berry-Esseen's inequality (Zolotarev (1967) gives the approximation 0.9051). Further,

$$
\left|\hat{\delta}_{n}-\sigma_{n}\right| \leqq C_{1}\left(|| \psi^{\prime}||+|| \psi^{\prime \prime}||\right) \Gamma_{n, 1}
$$

with an absolute constant $C_{1}$, provided $\psi^{\prime \prime}$ exists on $(0,1)$.

THEOREM 1.2. If $\psi$ has a second order derivative on $(0,1)$, then for any positive integers $n$ and $r$ such that $n^{-1} r^{3} \leqq \frac{3}{8}$,

$$
\begin{aligned}
\left\|H_{n}\left(\hat{\delta}_{n} \cdot\right)-\Phi(\cdot)\right\| \leqq & 4 C\left(2\|\psi\|^{3}+\left\|\psi^{\prime}\right\|^{3}\right) \sum_{i=1}^{n}\left|c_{i}\right|^{3} \hat{\delta}_{n}^{-3} \\
& +C_{2}\left[\hat{\delta}_{n}^{-1}\left(\left\|\psi^{\prime}\right\|+\left\|\psi^{\prime \prime}\right\|\right) r \Gamma_{n r}\right]^{2 r /(2 r+1)},
\end{aligned}
$$

where $C_{2}$ is an absolute constant.

REMARK. If the $c_{i}$ are chosen such that $\left|c_{i}\right| \leqq a / n^{\frac{1}{2}}$ with constant $a$ for all 
$i$ and $n$, then

$$
\Gamma_{n r} \leqq a / n^{\frac{1}{2}},
$$

and for $r=[\log n],\left[r \Gamma_{n r}\right]^{2 r /(2 r+1)} \leqq a \sqrt[4]{e}(\log n) n^{-\frac{1}{2}}(1+O(1 / \log n))$.

Note that $\hat{\delta}_{n}^{-1} c_{i}$ is invariant and thus also $\hat{\delta}_{n}{ }^{-1} \Gamma_{n r}$ is invariant under the transformation $c_{i} \rightarrow \gamma c_{i}, i=1,2, \cdots$.

2. Some lemmas.

LEMMA 2.1. For any positive integers $r$ and $n, 2 r \leqq n$, we have

$$
E\left[\left(\rho_{i}-\rho_{i i}\right)^{2 r}\right] \leqq b(r) n^{-r}
$$

with

$$
b(r) \leqq n^{-r} \sum_{t=1}^{r}\left(\begin{array}{c}
n-1 \\
t
\end{array}\right) \frac{(2 r) !}{(2 r-2 t) !} t^{2 r-2 t} \cdot 2^{-3 t}
$$

and for $n^{-1} r^{3} \leqq \frac{3}{4}$

$$
b(r) \leqq 2^{-3 r} \frac{(2 r) !}{r !}\left[1+8 n^{-1} r^{3}\right]
$$

Proof. By (1.4) we obtain

$$
\rho_{i}-\rho_{i i}=\frac{1}{n} \sum_{j \neq i}^{n}\left[u\left(X_{i}-X_{j}\right) F_{j}\left(X_{i}\right)\right] .
$$

By the polynomial theorem we then get

$$
\begin{array}{r}
E\left[\left(\rho_{i}-\rho_{i i}\right)^{2 r}\right]=n^{-2 r} \sum \frac{(2 r) !}{s_{1} ! \cdots s_{n} !} E \prod_{j \neq 1}^{n}\left[u\left(\bar{\wedge}_{i}-X_{j}\right)-F_{j}\left(X_{i}\right)\right]^{s_{j}}, \\
s_{1}+\cdots+s_{n}=2 r .
\end{array}
$$

We claim that any term in this sum is equal to zero if $s_{j_{0}}=1$ for some $j_{0}$. Indeed we find that the conditional expection of the product with respect to all $X_{j}, j \neq j_{0}$ is equal to 0 if $s_{j_{0}}=1$. Hence we have only to regard terms with $s_{j}=0$ or $\geqq 2$ for any $j$, and there can be at most $t \leqq r$ exponents $s_{j}$ different from 0 . If $s_{j} \geqq 2, j=1,2, \cdots, t, s_{j}=0$ for $j>t, i>t$ we obtain, observing that

$$
\begin{aligned}
& \left|u\left(X_{i}-X_{j}\right)-F_{j}\left(X_{i}\right)\right| \leqq 1 \\
& \begin{aligned}
E\left[\prod_{j=1}^{t}\left[u\left(X_{i}-X_{j}\right)-F_{j}\left(X_{i}\right)\right]^{s} j\right. & \leqq E \prod_{j=1}^{t}\left[u\left(X_{i}-X_{j}\right)-F_{j}\left(X_{i}\right)\right]^{2} \\
& =E\left[\prod_{j=1}^{t}\left[F_{j}\left(X_{i}\right)-F_{j}^{2}\left(X_{i}\right)\right]\right] \leqq 4^{-t} .
\end{aligned}
\end{aligned}
$$

This inequality remains true for all permutations of the indices $1, \cdots, n$. Put

$$
\gamma(t)=\sum_{8_{1}+\cdots+s_{t}=2 r ; 8_{j} \geq 2, j=1, \cdots, t} \frac{(2 r) !}{s_{1} ! \cdots s_{t} !} .
$$

Since $t$ indices out of $n-1$ indices can be chosen in $\left(\begin{array}{c}n-1 \\ t\end{array}\right)$ different ways we obtain from (2.4) through (2.6),

$$
E\left[\left(\rho_{i}-\rho_{i i}\right)^{2 r}\right] \leqq n^{-2 r} \sum_{t=1}^{r}\left(\begin{array}{c}
n-1 \\
t
\end{array}\right) \gamma(t) 4^{-t} .
$$


We claim that

$$
\gamma(t) \leqq \frac{(2 r) !}{(2 r-2 t) !} 2^{-t} t^{2 r-2 t}
$$

Indeed, differentiating the identity

$$
\left(\sum_{j=1}^{t} y_{j}\right)^{2 r}=\sum_{s_{1}+\cdots+s_{t}=2 r} \frac{(2 r) !}{s_{1} ! \cdots s_{t} !} \prod_{j=1}^{t} y_{j}^{s_{j}}
$$

twice with respect to all $y_{j}$ and then putting all $y_{j}$ equal to 1 , we obtain

$$
\frac{(2 r) !}{(2 r-2 t) !} t^{(2 r-2 t)}=\sum_{s_{1}+\cdots+s_{t}=2 r ; s_{j} \geq 2, j=1 \cdots t} \prod_{j=1}^{t} s_{j}\left(s_{j}-1\right) \frac{(2 r) !}{s_{1} ! \cdots s_{t} !} .
$$

Now using (2.7) and (2.8), we get (2.1) and (2.2). We now estimate $b(r)$ further, mainly for use when $n$ and $r$ are large. Put $r-t=u$. Then we can write

$$
b(r) \leqq 2^{-3 r} \sum_{u=0}^{r-1} k(u)
$$

with

Particularly

$$
k(u)=\frac{n^{-u}(2 r) !(r-u)^{2 u} 2^{3 u}}{(r-u) !(2 u) !}
$$

$$
k(0)=\frac{(2 r) !}{r !}, \quad k(1)<4 n^{-1} r^{3} \cdot \frac{(2 r) !}{r !}
$$

and for $u \geqq 1$

Hence

$$
\begin{aligned}
\frac{k(u+1)}{k(u)} & =n^{-1}\left(1-\frac{1}{r-u}\right)^{2 u} \cdot 2^{3} \cdot(r-u) \frac{(r-u-1)^{2}}{(2 u+1)(2 u+2)} \\
& <\frac{2}{3} n^{-1} r^{3} \leqq \frac{1}{2} \quad \text { for } \quad n^{-1} r^{3} \leqq \frac{3}{4} .
\end{aligned}
$$

$$
b(r) \leqq 2^{-3 r} \cdot \frac{(2 r) !}{r !}\left[1+8 n^{-1} r^{3}\right]
$$

for $n^{-1} r^{3} \leqq \frac{3}{4}$.

LEMMA 2.2. For any positive integers $r$ and $n, 2 r \leqq n$, we have

$$
E\left(T_{n}-\hat{T}_{n}\right)^{2 r} \leqq c(r)\|\psi \mid\|^{2 r} \Gamma_{n, r}^{2 r}
$$

if $\psi^{\prime}$ exists on $(0,1)$, and if $\psi^{\prime \prime}$ exists on $(0,1)$

$$
\begin{gathered}
E\left[\left(S_{n}-T_{n}\right)^{2 r}\right] \leqq b(2 r)\left\|\psi^{\prime \prime}\right\|^{2 r} \Gamma_{n, r}^{2 r}, \\
E\left[\left(S_{n}-\hat{T}_{n}\right)^{2 r}\right] \leqq d(r, \phi) \Gamma_{n, r}^{2 r}
\end{gathered}
$$

with

$$
\begin{aligned}
& b(2 r) \leqq n^{-2 r} \sum_{t=1}^{2 r}\left(\begin{array}{c}
n-1 \\
t
\end{array}\right) \frac{(4 r) !}{(4 r-2 t) !} t^{4 r-2 t} \cdot 2^{-3 t} \\
& c(r) \leqq 2^{2 r} n^{-2 r} \sum_{t=1}^{2 r}\left(\begin{array}{c}
n \\
t
\end{array}\right) \frac{(4 r) !}{(4 r-2 t) !} t^{4 r-2 t} \cdot 2^{-t} \\
& d(r, \phi) \leqq\left[[b(2 r)]^{1 / 2 r}\left\|\psi^{\prime \prime}\right\|+[c(r)]^{1 / 2 r}|| \psi^{\prime} \|\right]^{2 r}
\end{aligned}
$$


Further we have the estimates

$$
b(2 r) \leqq 2^{-6 r} \frac{(4 r) !}{(2 r) !}\left[1+2^{6} n^{-1} r^{3}\right]
$$

for $2^{3} n^{-1} r^{3} \leqq \frac{3}{4}$,

$$
c(r) \leqq \frac{(4 r) !}{(2 r) !}\left[1+2^{3} n^{-1} r^{3}\right] \quad \text { for } \quad n^{-1} r^{3} \leqq \frac{3}{8} .
$$

Remark. By Stirling's approximation of the $\Gamma$-function we have

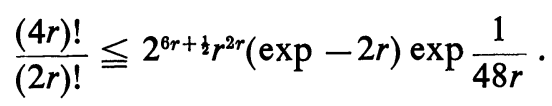

Proof. By (1.6) and (1.8) we get

$$
T_{n}-\hat{T}_{n}=\sum_{i=1}^{n} c_{i}\left\{\left(\rho_{i}-\rho_{i i}\right) \psi^{\prime}\left(\rho_{i i}\right)-\sum_{j=1 ; j \neq i}^{n} E\left[\left(\rho_{i}-\rho_{i i}\right) \psi^{\prime}\left(\rho_{i i}\right) \mid X_{j}\right]\right\}
$$
and for $j \neq i$

$$
\begin{aligned}
E\left[\left(\rho_{i}-\rho_{i i}\right) \psi^{\prime}\left(\rho_{i i}\right) \mid X_{j}\right] & =\frac{1}{n} \sum_{k \neq i}^{n} E\left\{\left[u\left(X_{i}-X_{k}\right)-F_{k}\left(X_{i}\right)\right] \psi^{\prime}\left(\rho_{i i}\right) \mid X_{j}\right\} \\
& \left.=\frac{1}{n} E\left[u\left(X_{i}-X_{j}\right)-F_{j}\left(X_{i}\right)\right] \psi^{\prime}\left(\rho_{i i}\right) \mid X_{j}\right],
\end{aligned}
$$

since the conditional expectations in the sum are zero for $j \neq k, i$. Now using the relation

$$
\left(\rho_{i}-\rho_{i i}\right) \psi^{\prime}\left(\rho_{i i}\right)=\frac{1}{n} \sum_{j \neq i}^{n}\left[u\left(X_{i}-X_{j}\right)-F_{j}\left(X_{i}\right)\right] \psi^{\prime}\left(\rho_{i i}\right),
$$

and noting that

$$
E\left[\left(\rho_{i}-\rho_{i i}\right) \psi^{\prime}\left(\rho_{i i}\right) \mid X_{i}\right]=0
$$

we obtain from $(2.15)$

$$
T_{n}-\hat{T}_{n}=\frac{1}{n} \sum_{i=1}^{n} \sum_{j \neq i}^{n} c_{i} V_{i j}
$$

with

Clearly

$$
\begin{aligned}
V_{i j}=\left[u\left(X_{i}-X_{j}\right)-F_{j}\left(X_{i}\right)\right] \psi^{\prime}\left(\rho_{i i}\right) & \\
& -E\left\{\left[u\left(X_{i}-X_{j}\right)-F_{j}\left(X_{i}\right)\right] \psi^{\prime}\left(\rho_{i i}\right) \mid X_{j}\right\} .
\end{aligned}
$$

$$
E\left[V_{i j} \mid X_{j}\right]=0, \quad E\left[V_{i j} \mid X_{i}\right]=0 .
$$

By the polynomial theorem we get

$$
\begin{aligned}
E\left[\left(T_{n}-\hat{T}_{n}\right)^{2 r}\right] & =n^{-2 r} E\left[\sum_{i=1}^{n} \sum_{j \neq i}^{n} c_{i} V_{i j}\right]^{2 r} \\
& =n^{-2 r} \sum \frac{(2 r) !}{\prod_{i=1}^{n} \prod_{j \neq i}^{n}\left(s_{i j} !\right)} E\left\{\prod_{i=1}^{n} \prod_{j \neq i}^{n}\left(c_{i} V_{i j}\right)^{s_{i j}}\right\}
\end{aligned}
$$

where the sum should be taken over terms corresponding to different vector solutions $\left\{s_{i j}\right\}, i, j=1, \cdots n, j \neq i$ of the equation

$$
\sum_{i=1}^{n} \sum_{j \neq i}^{n} s_{i j}=2 r \text {. }
$$


The expectation

$$
E\left[\prod_{i=1}^{n} \prod_{j \neq i}^{n} V_{i j}{ }^{s_{i j}}\right]
$$

is equal to 0 for some vector solutions of (2.21) since (2.19) holds, and we have only to regard those solutions for which the expectation (2.22) is not equal to 0 .

We say that $s_{i j}$ gives the contribution $\frac{1}{2} s_{i j}$ to the sum (2.21) from each of the indices $i$ and $j$. Hence according to this notation an index $k$ gives the contribution

$$
g(k)=\frac{1}{2} \sum_{j \neq k}^{n} s_{k j}+\frac{1}{2} \sum_{j \neq k}^{n} s_{j k}
$$

to the sum (2.21). By conditioning with respect to all $X_{j}, j \neq k$ we easily find that the expectation (2.22) is equal to 0 if $k$ gives the contribution $\frac{1}{2}$ to the sum (2.21), i.e., if $s_{k j}=1$ for exactly one index $j \neq k$, and $s_{j k}=0$ for $j \neq k$ or if $s_{j k}=1$ for exactly one $j$ and $s_{k j}=0$ for $j \neq k$.

The sum $\sum$ on the right-hand side of $(2.20)$ can be divided into partial sums as follows. Let $C$ be a collection of different positive integers belonging to the set $1, \cdots, 2 r$, say $C=(1,2, \cdots, t)$. Let $\sum_{C}$ consist of all terms in (2.20) corresponding to the vector solutions of (2.21) such that

(a) $s_{i j}=0$ if not both $i$ and $j$ belong to $C$;

(b) for any $k \in C$ the contribution to the sum (2.21) is larger than $\frac{1}{2}$. Note that $C$ can contain at most $2 \mathrm{r}$ different integers since every $k \in C$ gives at least the contribution 1 to the sum (2.21). Clearly partial sums $\sum_{C_{1}}$ and $\sum_{C_{2}}$ contain no common terms if $C_{1} \neq C_{2}$. Consider now the expectation

$$
E\left[\prod_{i=1}^{t} \prod_{j \neq i}^{t}\left(c_{i} V_{i j}\right)^{s_{i j}}\right]
$$

where the $i$ and $j$ belong to the collection $C$. Note that $s_{i j}$ may be equal to 0 for some pairs $(i, j)$. By Hölder's inequality we get, using the fact that $\left|V_{i j}\right| \leqq$ $2\left\|\psi^{\prime}\right\|$,

where

$$
\begin{aligned}
\left|E \prod_{i=1}^{t} \prod_{j \neq i}^{t}\left(c_{i} V_{i j}\right)^{s_{i i}}\right| & \leqq \prod_{i=1}^{t} \prod_{j \neq i}^{t}\left|c_{i}\right|^{s_{i} j}\left\{E\left[\left(V_{i j}\right)^{2 r}\right]\right\}^{s_{i j} / 2 r} \\
& \leqq 2^{2 r}|| \psi^{\prime}||^{2 r} \prod_{i=1}^{t}\left|c_{i}\right|^{s_{i}}
\end{aligned}
$$

The partial sum corresponding to $C$ is then estimated by

$$
\sum_{c}^{\prime} \frac{(2 r) !}{\prod_{i=1}^{t} \prod_{i \neq j}^{t}\left(s_{i j}\right) !}\left(2^{2 r}|| \psi^{\prime} \|^{2 r} \prod_{i=1}^{t}\left|c_{i}\right|^{s_{i}}\right) .
$$

Note that $(2 r) ! / \prod_{i=1}^{t} \prod_{i \neq j}^{t}\left(s_{i j}\right) !$ is an integer. Hence we have

$$
N(t)=\sum_{c}^{\prime} \frac{(2 r) !}{\prod_{i=1}^{t} \prod_{j \neq i}^{t}\left(s_{i j}\right) !}
$$

terms in the class $C$ which are estimated by (2.24). Let $\mathscr{C}_{t}$ be the set of all terms

$$
\sum \prod_{i=1}^{n} \prod_{j \neq 1}^{n}\left(c_{i} V_{i j}\right)^{s_{i j}}
$$


in (2.23) which belong to some class $C$ containing exactly $t$ indices. Let $\left(s_{1}\right.$, $\left.s_{2}, \cdots, s_{t}\right)$ in (2.26) be given, $0 \leqq s_{1} \leqq s_{2}<\cdots \leqq s_{t}, \sum_{i=1}^{t} s_{i}=2 r$. Then according to the symmetry the set $\mathscr{C}_{t}$ contains a sum of terms, each estimated by

$$
2^{2 r}\left\|\psi^{\prime}\right\|^{2 r} \prod_{i=1}^{t}\left|c_{k_{i}}\right|^{s_{i}}
$$

where $\left(k_{1} \cdots k_{t}\right)$ is any combination of numbers $1,2, \ldots, n$ to the $t$ th class and in any order within this class. Let the number of terms in $C_{t}$ for a fixed vector $\left(s_{1}, s_{2}, \cdots, s_{t}\right)$ as above be $n(t)$ and the sum of terms (2.27) belonging to $\left(s_{1}\right.$, $\left.s_{2}, \cdots, s_{t}\right)$ be $A\left(s_{1}, s_{2}, \cdots, s_{t}\right)$. (Note that $n(t)$ depends on $s_{1}, \cdots, s_{t}$. ) Then, since $A\left(s_{1}, \cdots, s_{t}\right)$ is a symmetrical function

$$
A\left(s_{1}, s_{2}, \cdots, s_{t}\right)=\frac{n(t)}{n !} \sum^{\prime 2 r}\left\|\psi^{\prime}\right\|^{2 r} \prod_{i=1}^{t_{i}}\left|c_{k_{i}}\right|^{s_{i}}
$$

where $\Sigma^{\prime}$ is the sum all terms belonging to all permutations of the numbers $1,2, \cdots, n$. By Hölder's inequality we get, observing that

$$
\begin{gathered}
\left|c_{k_{i}}\right|^{s_{i}}=\left[c_{k_{i}}^{2 r}\right]^{s_{i} / 2 r}, \quad \sum_{i=1}^{t} \frac{s_{i}}{2 r}=1, \\
\sum^{\prime} \prod_{i=1}^{t}\left|c_{k_{i}}\right|^{s_{i}} \leqq \prod_{i=1}^{t}\left(\Sigma^{\prime} c_{k_{i}}^{2 r} i^{s_{i} / 2 r}\right.
\end{gathered}
$$

and here

$$
\sum^{\prime} c_{k_{i}}^{2 r}=\frac{n !}{n} \sum_{i=1}^{n} c_{i}^{2 r}
$$

Hence we obtain by $(2.28)$ and $(2.29)$

$$
A\left(s_{1}, s_{2}, \cdots, s_{t}\right) \leqq 2^{2 r}\left\|\psi^{\prime}\right\|^{2 r} \cdot n(t) \cdot \frac{1}{n} \sum_{i=1}^{n} c_{i}^{2 r} .
$$

Since $\mathscr{C}_{t}$ contains $\left(\begin{array}{l}n \\ t\end{array}\right) N(t)$ terms we then find that $\mathscr{C}_{t}$ gives at most the contribution

$$
n^{-2 r_{2} 2 r}\left\|\psi^{\prime}\right\|^{2 r}\left(\begin{array}{l}
n \\
t
\end{array}\right) N(t) \cdot \frac{1}{n} \sum_{i=1}^{n} c_{i}^{2 r}
$$

to the right-hand side of (2.20). Putting

$$
\Gamma_{n r}^{2 r}=\frac{1}{n} \sum_{i=1}^{n} c_{i}^{2 r}, \quad \Gamma_{n r} \geqq 0,
$$

and regarding the sets $\mathscr{C}_{t}$ for $t=1,2, \ldots, 2 r$, we obtain from (2.20) that

$$
E\left[\left(T_{n}-\hat{T}_{n}\right)^{2 r}\right] \leqq 2^{2 r} n^{-2 r}\left\|\psi^{\prime}\right\|^{2 r} \Gamma_{n r}^{2 r} \sum_{t=1}^{2 r}\left(\begin{array}{l}
n \\
t
\end{array}\right) N(t) .
$$

We estimate $N(t)$ in the following way. Consider the identity

$$
\left(\sum_{i=1}^{t} \sum_{j=i}^{t} x_{i} x_{j}\right)^{2 r}=\sum \frac{(2 r) !}{\prod_{i=1}^{t} \prod_{j=1}^{t}\left(s_{i j}\right) !} \prod_{i=1}^{t} \prod_{j \neq i}^{t}\left(x_{i} x_{j}\right)^{s_{i j}} .
$$

If an index $k$ gives the contribution $\geqq 1$ to the sum (2.21), i.e., to the sum

$$
\sum_{i=1}^{t} \sum_{j \neq i}^{t} s_{i j}=2 r,
$$


then the double product

$$
\prod_{j=1}^{t} \prod_{j \neq i}^{t}\left(x_{i} x_{j}\right)^{s_{i j}}
$$

contains $x_{k}$ as factor at least in the power 2 . Hence differentiating the identity twice with respect to each $x_{k}, k=1,2, \ldots, t$ and then putting all $x_{n}$ equal to 1 we get the inequality

$$
2^{t} N(t) \leqq\left\{\prod_{k=1}^{t} \frac{\partial^{2}}{\partial_{x_{k}}}\left(\sum_{i=1}^{t} \sum_{j \neq i}^{t} x_{i} x_{j}\right)^{2 r}\right\}_{x_{k}=1, k=1,2, \cdots, t} .
$$

The right-hand side, however, is at most equal to

$$
\left\{\prod_{k=1}^{t} \frac{\partial^{2}}{\partial x_{k}}\left(\left(\sum_{i=1}^{t} x_{i}\right)^{4 r}\right)\right\}_{x_{k}=1, k=1, \cdots, t}=\frac{(4 r) !}{(4 r-2 t) !} t^{4 r-2 t} .
$$

Combining (2.30), (2.32) and (2.33), we get

with

$$
E\left[\left(T_{n}-\hat{T}_{n}\right)^{2 r}\right] \leqq c(r)\left\|\psi^{\prime}\right\|^{2 r} \Gamma_{n r}^{2 r}
$$

$$
\begin{aligned}
& c(r)=2^{2 r} n^{-2 r} \sum_{t=1}^{2 r}\left(\begin{array}{l}
n \\
t
\end{array}\right) \frac{(4 r) !}{(4 r-2 t) !} t^{4 r-2 t} \cdot 2^{-t} \\
& \Gamma_{n r}^{2 r}=\frac{1}{n} \sum_{i=1}^{n}\left|c_{i}\right|^{2 r} .
\end{aligned}
$$

We estimate $c(r)$ exactly in the same way as we have estimated $b(r)$ in Lemma 2.1 and then obtain for $u=2 r-t$

with

$$
c(r) \leqq \sum_{u=0}^{r-1} k(u)
$$

$$
k(u)=n^{-u} \frac{(4 r) !}{(2 u) !(2 r-u) !}(2 r-u)^{2 u} \cdot 2^{u} .
$$

Hence

and for $u \geqq 1$

$$
k(0)=\frac{(4 r) !}{(2 r) !}, \quad k(1)<n^{-1} \cdot(2 r)^{3} \frac{(4 r) !}{(2 r) !}
$$

$$
\frac{k(u+1)}{k(u)} \leqq \frac{4}{3} n^{-1} r^{3} \leqq \frac{1}{2} \quad \text { for } \quad n^{-1} r^{3} \leqq \frac{3}{8} .
$$

Hence for $n^{-1} r^{3} \leqq \frac{3}{8}$

$$
c(r) \leqq \frac{(4 r) !}{(2 r) !}\left[1+8 n^{-1} r^{3}\right] .
$$

Thus we have proved (2.13) and (2.14) of the lemma.

It follows by the definition of $T_{n}$ that

with

$$
S_{n}-T_{n}=\sum_{i=1}^{n} c_{i}\left[\xi_{i}-E\left(\xi_{i}\right)\right]
$$

$$
\left|\xi_{i}\right| \leqq \frac{1}{2}\left(\rho_{i}-\rho_{i i}\right)^{2}|| \psi^{\prime \prime} \|
$$


Hence

$$
E\left[\left(S_{n}-T_{n}\right)^{2 r}\right] \leqq n^{2 r-1} \sum_{i=1}^{n} c_{i}{ }^{2 r} E\left[\left(\xi_{i}-E \xi_{i}\right)^{2 r}\right]
$$

and by Lemma 2.1

$$
E\left[\left(\xi_{i}-E\left(\xi_{i}\right)\right)^{2 r}\right] \leqq 2^{2 r} E\left[\xi_{i}{ }^{2 r}\right] \leqq\left\|\psi^{\prime \prime}\right\|^{2 r} E\left[\left(\rho_{i}-\rho_{i i}\right)^{4 r}\right] \leqq n^{-2 r} b(2 r)\left\|\phi^{\prime \prime}\right\|^{2 r} .
$$

Thus we get (2.11)

$$
E\left[\left(S_{n}-T_{n}\right)^{2 r}\right] \leqq b(2 r) \Gamma_{n r} .
$$

By Minkovski's inequality we obtain (2.12) from (2.10) and (2.11)

$$
E^{1 / 2 r}\left[\left(S_{n}-\hat{T}_{n}\right)^{2 r}\right] \leqq E^{1 / 2 r}\left[\left(S_{n}-T_{n}\right)^{2 r}\right]+E^{1 / 2 r}\left[\left(T_{n}-\hat{T}_{n}\right]^{2 r}\right]
$$

Lemma 2.3. $\hat{T}_{n}=\sum_{j=1}^{n} \hat{T}_{n}^{(j)}$ with independent random variables

$$
\begin{aligned}
\hat{T}_{n}^{(j)}=c_{j}\left\{\phi\left(\rho_{j j}\right)-E\left[\psi\left(\rho_{j j}\right)\right]\right\} \\
\quad+\frac{1}{n} \sum_{i \neq j}^{n} c_{i}\left[E\left(u\left(X_{i}-X_{j}\right)-F_{j}\left(X_{i}\right)\right) \psi^{\prime}\left(\rho_{i i}\right) \mid X_{j}\right] .
\end{aligned}
$$

Further,

(ii)

$$
\sum_{j=1}^{n}\left[E\left|\hat{T}_{n}^{(j)}\right|^{3}\right] \leqq 4\left[2\|\psi \mid\|^{3}+\left\|\psi^{\prime}\right\|^{3}\right\} \sum_{j=1}^{n}\left|c_{i}\right|^{3} .
$$

Proof. We get the representation (i) by (2.16). Using well-known inequalities

$$
\left|(a+b)^{3}\right| \leqq 4\left[|a|^{3}+|b|^{3}\right], \quad\left|\left(\sum_{i=1}^{n} a_{i}\right)^{3}\right| \leqq n^{2} \sum_{i=1}^{n}\left|a_{i}\right|^{3}
$$

we obtain

$$
E\left[\left|\hat{T}_{n}^{(j)}\right|^{3}\right] \leqq 4\left|c_{j}\right|^{3} E\left[\left|\left[\psi\left(\rho_{j j}\right)\right]-E \psi\left(\rho_{j j}\right)\right|^{3}\right]+\frac{4}{n} \sum_{i \neq j}^{n}\left|c_{i}\right|^{3}\left\|\psi^{\prime}\right\|^{3} .
$$

Here

$$
E\left[\left|\psi\left(\rho_{j j}\right)-E\left[\psi\left(\rho_{j j}\right)\right]\right|^{3}\right] \leqq 2\|\psi\| E\left(\psi\left(\rho_{j j}\right)-E\left(\psi\left(\rho_{j j}\right)\right)^{2} .\right.
$$

Thus we get (ii).

\section{Proofs of the theorems.}

(a) Proof of Theorem 1.1. (1.10) follows from Berry-Esseen's inequality and Lemma 2.3 and (1.11) from Lemma 2.2 (2.12).

(b) Proof of Theorem 1.2. For $h>0$ we get

$$
\begin{aligned}
P\left[S_{n} \leqq \hat{\delta}_{n} x\right] & \leqq P\left(S_{n} \leqq \hat{\delta}_{n} x,\left|S_{n}-\hat{T}_{n}\right|<h \hat{\delta}_{n}\right)+P\left[\left|S_{n}-\hat{T}_{n}\right| \geqq h \delta_{n}\right] \\
& \leqq P\left[\hat{T}_{n} \leqq \hat{\delta}_{n}(x+h)\right]+P\left[\left|S_{n}-\hat{T}_{n}\right| \geqq h \hat{\delta}_{n}\right]
\end{aligned}
$$

Applying Theorem 1.1 we get

$$
P\left[\hat{T}_{n} \leqq \hat{\delta}_{n}(x+h)\right] \leqq \Phi(x+h)+4 C\left(2\|\psi\|^{3}+\left\|\psi^{\prime}\right\|^{3}\right) \cdot \sum_{i=1}^{n}\left|c_{i}\right|^{3} \hat{\delta}_{n}^{-3} .
$$

Here

$$
\Phi(x+h) \leqq \Phi(x)+\left\|\Phi^{\prime}(x)\right\|=\Phi(x)+\frac{h}{(2 \pi)^{\frac{1}{2}}} .
$$

By Chebyshev's inequality and the inequality (2.12) of Lemma 2.2 we get

$$
P\left[\left|S_{n}-\hat{T}_{n}\right| \geqq h \hat{\delta}_{n}\right] \leqq d(r, \phi) \Gamma_{n r}^{2 r}\left(h \hat{\delta}_{n}\right)^{-2 r} .
$$


Now we choose $n$ such that

i.e.,

$$
\frac{h}{(2 \pi)^{\frac{1}{2}}}=d(r, \phi) \Gamma_{n r}^{2 r}\left(h \hat{\delta}_{n}\right)^{-2 r}
$$

It follows by Lemma 2.2, (2.12), (2.13) and (2.14), and the remark made after Lemma 2.2 that for $n^{-1} r^{3} \leqq \frac{3}{8}$

$$
[d(r, \psi)]^{1 / 2 r} \leqq C^{\prime} r\left(\left\|\psi^{\prime}\right\|+\left\|\psi^{\prime \prime}\right\|\right)
$$

with an absolute constant $C^{\prime}$. Then it follows by (3.4) and (3.5) that

$$
\frac{h}{(2 \pi)^{\frac{1}{2}}}+d(r, \psi) \Gamma_{n r}^{2 r}\left(h \hat{\delta}_{n}\right)^{-2 r} \leqq C_{2}\left[\hat{\delta}_{n}^{-1}\left(\left\|\psi^{\prime}\right\|+\left\|\psi^{\prime \prime}\right\|\right) r \Gamma_{n r}\right]^{2 r / 2 r+1)}
$$

By (3.1)-(3.6) we get the inequality (1.12) in one direction. It follows for the other direction in the same way.

Acknowledgment. The authors would like to express their sincere appreciation to Professor I. R. Savage, the associate editor, and the referees for the critical examination of the original draft. Their constructive criticism, detailed comments and suggestions for improvements are gratefully acknowledged.

\section{REFERENCES}

[1] Hájek, J. (1962). Asymptotically most powerful rank order tests. Ann. Math. Statist. 33 $1124-1147$.

[2] Hájek, J. (1968). Asymptotic normality of simple linear rank statistics under alternatives. Ann. Math Statist. 39 325-346.

[3] Hájek, J. and Šidák, Z. (1967). Theory of Rank Tests. Academic Press, New York.

[4] Jurečková, J. and Puri, Madan L. (1975). Order of normal approximation for rank test statistics distribution. Ann. Probability 3 526-533.

[5] Zolotarev, V. M. (1967). A sharpening of the inequality of Berry-Esseen. Z. Wahrscheinlichkeitstheorie und Verw. Gebiete 8 332-342.

Department of Mathematics INDIANA UNIVERSITY

Swain Hall, East

BLOOMINGTON, IN 47401 